\title{
Dosage rapide de la matière grasse des fromages
}

\author{
par \\ M. DESFLEURS \\ Ingénieur chimiste I.C.L. Docteur ès-sciences (1) \\ et B. BAILLEUL \\ Directeur du Laboratoire Interprofessionnel \\ laitier du Calvados (2)
}

La méthode de référence pour le dosage de la matière grasse des fromages est celle de Schmid-Bondzynski-Ratzlaff, dite S.B.R. [4] qui n'est qu'une modification de la méthode Röse-Gottlieb [9] substituant l'acide chlorhydrique à l'ammoniaque comme dissolvant. Elle est longue et onéreuse ; aussi les fromageries utilisent généralement le procédé acido-butyrométrique dit de Gerber [2] avec des butyromètres de Van Gülick.

Remarquons tout d'abord que la graduation des butyromètres Van Gülick est basée sur une densité de la matière grasse qui est la même que celle utilisée pour les butyromètres à lait [5].

On peut, en effet, très bien se servir de ces derniers pour doser la matière grasse des fromages, en suivant par exemple le mode opératoire de Siegfeld. Il consiste à dissoudre environ $3 \mathrm{~g}$ de fromage exactement pesés par de l'acide sulfurique de densité 1,60 , en chauffant dans un bécher. On verse la solution dans un butyromètre. On rince avec l'acide et complète de manière à avoir $21 \mathrm{ml}$. On ajoute alors $1 \mathrm{ml}$ d'alcool isoamylique et l'on termine comme usuel. Le nombre de petites graduations lues multiplié par 1,1 et divisé par le poids pesé donne le pourcentage de matière grasse. En effet, chaque petite division correspond à $0,011 \mathrm{~g}$ de matière grasse. C'est pourquoi nous avons substitué 1,1 au coefficient 1,133 indiqué par Siegfeld, qui ne se justifie pas.

Il est possible également, comme nous le faisons, de peser exactement $3 \mathrm{~g}$ de fromage, soit directement dans le butyromètre à lait, soit dans une feuille de papier à cigarette non gommée que l'on roule pour l'introduire dans le butyromètre. On ajoute alors environ

(1) Institut du Lait, des Viandes et de la Nutrition à Caen.

(2) Laboratoire Interprofessionnel Laitier du Calvados à Herouville-Saint-Clair. 
$18,5 \mathrm{ml}$ d'acide sulfurique de densité 1,525 . On s'aide d'un séjour au bain-marie à $70^{\circ} \mathrm{C}$ pour faciliter la dissolution. Quand elle est complète, on verse $1 \mathrm{ml}$ d'alcool isoamylique et l'on poursuit comme d'habitude. Le mode de calcul est le même. L'on trouve les mêmes résultats qu'avec le butyromètre Van Gülick, toutefois avec une meilleure approximation, la graduation étant plus fine.

Les laboratoires dotés d'un lecteur automatique et électronique des butyromètres Gerber, comme c'est le cas du Laboratoire Interprofessionnel Laitier du Calvados, peuvent ainsi faire les lectures avec une bien meilleure précision. Celles-ci sont exprimées en 1/10 de gramme avec une très bonne reproductibilité.

On pourrait aussi utiliser les butyromètres à poudre de lait suivant Teichert [14] mais la graduation ne permet pas une lecture aussi précise. En pratinue courante, on se sert du procédé Van Gülick pour plus de simplicité.

Si l'on compare les résultats du Van Gülick et du S.B.R. on constate une bonne coïncidence si l'on analyse des fromages frais venant d'être démoulés avec, à l'évidence, concordance avec la matière grasse «Gerber » du lait mis en œuvre. Par contre, avec des fromages affinés, il y a un écart d'autant plus grand que le fromage est plus maturé, les chiffres Van Gülick étant par excès. En exemple, voici les résultats trouvés sur des Camemberts entièrement affinés :

\begin{tabular}{l|l|l|l|l}
\hline S.B.R. matière grasse (p. 100) & 17,3 & 14,55 & 17,1 & 21,6 \\
Van Gülick matière grasse (p. 100) & 18,7 & 15,95 & 19,5 & 22,5 \\
\hline
\end{tabular}

Pour confirmer ces résultats, nous avons introduit dans un butyromètre à lait et pesé exactement $0,353 \mathrm{~g}$ de matière grasse d'un Camembert affiné, extraite par la S.B.R. Nous avons ajouté $11 \mathrm{ml}$ d'eau, $10 \mathrm{ml}$ d'acide sulfurique, $1 \mathrm{ml}$ d'alcool isoamylique et centrifugé. A $65^{\circ} \mathrm{C}$ la matière gasse occupe 34,5 petites divisions.

$$
\text { Or, nous n'aurions dû en trouver que } \frac{0,353}{0,011}=32,09 \text { soit } 32 \text {. Cette }
$$

augmentation se situe parmi celles que donnent, par rapport aux S.B.R. les analyses Van Gülick que nous avons citées plus haut.

Quelles en sont les raisons?

On sait que la matière grasse isolée par la méthode acidobutyrométrique n'est pas pure, ni même entièrement séparée. P. Cador et P. Macherel [3] admettent même une sulfonation en plus d'une certaine hydrolyse des triglycérides libérant des acides gras. Nous avons trouvé que ces acides gras forment des esters d'isoamyle.

En 1942-1943, la pénurie d'alcool isoamylique fit conseiller sa récupération par distillation des résidus de l'analyse Gerber. Nous 
constatâmes nous aussi [10] que l'alcool brut que nous avions ainsi obtenu ne donnait pas un résultat nul lors de l'essai à blanc. Il fallait effectuer une seconde distillation entre $128^{\circ} \mathrm{C}$ et $132^{\circ} \mathrm{C}$ sur colonne de Vigreux pour obtenir un produit utilisable.

Dans ces années de pénurie, le Laboratoire Interprofessionnel Laitier du Pays d'Auge s'approvisionna longtemps de cette manière. L'un de nous alors, en s'aidant du froid, sépara la matière grasse solidifiée surnageante du liquide sous-jacent, lequel distillé, fournit un alcool récupéré dont l'essai à blanc n'indiquait aucune matière grasse.

Prenant alors cette graisse solidifiée prise à la surface, après l'avoir lavée pour enlever l'acide, il la soumit à l'entraînement par la vapeur d'eau. Il recueillit à l'aide d'un vase florentin, un liquide d'odeur agréable, insoluble dans l'eau et moins dense qu'elle, constitué principalement par des esters d'isoamyle.

Nous soulignons que dans les expériences ci-dessus, il s'agissait de résidus Gerber d'analyses de laits.

Des études récentes [8] ont confirmé qu'il y a bien hydrolyse des triglycérides et estérification des acides gras libérés, ces phénomènes s'accentuant avec la durée de chauffage [11].

Ceci explique ce que nous disions plus haut au sujet de l'analyse des fromages frais, venant d'être démoulés. La matière est la même que celle du lait et l'on peut dire que le traitement est équivalent dans le butyromètre Van Gülick et dans le butyromètre Gerber. Les résultats sont justes puisque ces butyromètres gradués par comparaison en tiennent compte.

A l'évidence il n'en est pas de même pour les fromages affinés. Il y a lipolyse au cours de la maturation et d'autant plus qu'elle est plus avancée [13, 15]. Il y a formation également d'acides volatils par fermentation [7]. Tous ces acides aliphatiques s'ajoutant à ceux formés par hydrolise au cours de l'analyse seront susceptibles de s'estérifier et majoreront le résultat trouvé.

Nous pouvons dire également que la matière grasse extraite des fromages n'a pas la même composition que celle du lait pour laquelle les butyromètres sont gradués.

J. Pien et Mlle Maurice [12] ont montré que la densité de la matière grasse de produits fermentés est supérieure à celle provenant de lait frais. Comme on lit un volume et que l'on exprime un poids, on devrait donc obtenir avec les fromages un chiffre trop faible. C'est le contraire qui se produit, car l'influence de la variation de densité est de peu d'importance par rapport à l'estérification.

Il résulte de tout ceci que l'application d'un facteur de correction n'est pas possible. Il devrait varier avec la nature du fromage, son degré de maturation, la température de chauffage et sa durée.

L'obtention de résultats trop forts par la méthode acido-butyrométrique n'est pas sans inconvénients. Elle peut inciter les fromagers 
à une fausse sécurité quant au pourcentage de matière grasse par rapport à la matière sèche qu'ils doivent garantir dans leurs fromages, et par conséquent s'exposer à des poursuites par le Service de la Répression des Fraudes. Aussi généralement, ils emprésurent un lait plus riche qu'il ne serait strictement nécessaire, même en tenant compte des marges de sécurité.

Pour mettre en évidence, dans la pratique, l'augmentation de la matière grasse que donne la méthode Van Gülick, prenons une fabrication de Camemberts de lait cru dans une usine qui contrôle par cette technique d'analyse environ 25 fabrications par mois. Examinons par exemple la moyenne de 15 mois, exactement celle de 378 analyses. Par détermination sur les Camemberts venant d'être salés $\left(3^{e} j\right)$ on obtient la moyenne de : poids $311,1 \mathrm{~g}$, matière grasse p. 10017,9 , soit une matière grasse totale de $55,7 \mathrm{~g}$.

Pour les mêmes fabrications sur les Camemberts en début d'affinage au $16^{\mathrm{e}} \mathrm{j}$, on trouve, toujours en moyenne : poids $279,2 \mathrm{~g}$, matière grasse 20,4 p. 100 , soit une matière grasse totale de $56,95 \mathrm{~g}$.

A l'évidence la matière grasse n'a pas augmenté. On nous objectera que les analyses n'ont pas porté sur le même fromage, la seconde étant faite sur un autre Camembert de la même fabrication. C'est pourquoi nous avons pris la moyenne de 378 résultats, afin que les différences puissent se compenser.

Envisageons maintenant un cas constitué par trois échantillons de Camemberts remis aux experts, lors de poursuite pour présomption de fraude, plus de 8 mois après leur prélèvement.

Les mauvaises conditions de conservation ont fait que les fromages sont altérés et liquéfiés. L'ouverture des flacons provoque un dégagement d'ammoniac, de gaz à odeur putride et rance. La fermentation s'est poursuivie malgré l'adjonction de formol et avec elle la lipolyse, puisque sur la matière grasse de l'un des échantillons isolée par la S.B.R. le tirage indique une acidité de 82,5 p. 100 exprimée en acide oléïque.

Les experts jugent inutile de faire des analyses qui ne peuvent donner aucun reflet de la composition primitive. Néanmoins, déterminons en la matière grasse par trois méthodes différentes. Les chiffres ci-dessous sont la moyenne de deux analyses :

\begin{tabular}{l|c|c|c}
\hline Matière grasse p. 100 Van Gülick & 19,3 & 19,5 & 20,1 \\
Matière grasse p. 100 éthéro-chlorhydrique & 17,1 & 16,7 & 17,2 \\
Matière grasse p. 100 S.B.R. & 17,9 & 19,0 & 18,0 \\
\hline
\end{tabular}

Nous constatons : - Que la Van Gülick donne des résultats par excès pour les raisons que nous avons énoncées. - Que l'extrac- 
tion éthero-chlorhydrique [2], référence pour le lait, mais appliquée au fromage, donne des pourcentages par défaut. Cela est dû à l'élimination partielle ou totale des acides aliphatiques solubles dans l'eau lors de la filtration de la dissolution chlorhydrique et des lavages des filtres à l'eau bouillante jusqu'à disparition de l'ion chlore. - Que la S.B.R. paraît être la plus appropriée, bien qu'il faille prendre des précautions lors de l'évaporation des éthers. Nous la faisons sur une plaque chauffante réglée à $60^{\circ} \mathrm{C}$, température maximale d'ébullition de l'éther de pétrole utilisé, sous une hotte bien ventilée.

Si l'on peut très bien se servir des butyromètres Mojonnier pour la séparation de la couche aqueuse, il ne faut pas évaporer les éthers avec l'appareil de cette marque qui est constitué d'unf plaque chauffante à $135^{\circ} \mathrm{C}$ sous ventilation. Par exemple, pour une matière grasse, certes hydrolisée, de 20,4 p. 100 trouvée par évaporation ménagée, on n'a plus que 18,6 p. 100 avec l'appareil Mojonnier. Dans la S.B.R. il faut donc évaporer le mélange des éthers lentement, à la température la plus basse possible, si l'on ne veut pas avoir des résultats par défauts, dus à l'entraînement des acides gras volatils, lors de l'analyse des fromages affinés, d'autant plus que l'on peut craindre une certaine hydrolyse durant la dissolution par l'acide chlorhydrique concentré pendant $20 \mathrm{mn}$ au bain-marie bouillant.

Pour une analyse rapide, nous avons pensé que la principale source de divergences dans la méthode acido-butyrométrique est l'estérification. Nous nous sommes donc orientés vers un procédé n'utilisant pas d'alcool isoamylique ou autre et nous avons expérimenté celui décrit par E. Heiss [6]. Nous l'avons amendé et l'appliquons comme suit :

La dissolution de la pâte de fromage est faite à l'aide d'un mélange à volume égal d'acide perchlorique à 60 p. 100 et d'acide acétique glacial technique, avec mise au bain-marie à $70^{\circ} \mathrm{C}$. On utilise le butyromètre Van Gülick après avoir pesé très exactement $3 \mathrm{~g}$ de fromage. La dissolution est plus facile; elle est plus rapide. Il faut faire après dissolution l'ajustement du niveau des liquides jusqu'au trait 35 de la colonne du butyromètre avec de l'eau distillée chaude. On fait deux centrifugations de $5 \mathrm{mn}$ à $1100 \mathrm{t} / \mathrm{mn}$. La lecture est grandement facilitée par l'absence totale de dépôt noir entre la matière grasse et le liquide acide sous-jacent. Malheureusement les réactifs sont plus chers que l'acide sulfurique d : 1,525 employé dans la méthode Van Gülick, bien que l'absence d'alcool isoamylique dans la méthode Heiss fasse baisser le coût des produits. Nous avons pensé que l'on pouvait aussi modifier la composition du mélange des acides et nous avons obtenu de très bons résultats avec le mélange suivant :

Acide acétique

Acide perchlorique

40 p. 100

Acide chlorhydrique pur

40 p. 100

20 p. 100 
On améliore encore la dissolution qui peut se faire à $60^{\circ} \mathrm{C}$ et le coût du mélange est abaissé.

Bien entendu, on peut utiliser le butyromètre à lait suivant la technique que nous avons indiquée, avec environ $20 \mathrm{ml}$ du mélange des trois acides et $3 \mathrm{~g}$ de fromage.

Par exemple, pour un même fromage, les pourcentages de matière grasse sont:

Heiss, mélange des deux acides, butyromètre Van Gülick

22,49 et 22,75

22,12 et 22,04

22,72 et 22,85

22,76 et 22,80 moy. 22,62

moy. 22,08

moy. 22,79

moy. 22,78

Van Gülick, butyromètre à lait

Voici maintenant quelques résultats obtenus en utilisant pour les méthodes Van Gülick et Heiss les butyromètres Van Gülick, c'est-àdire avec une approximation de lecture de 0,5 p. 100 au plus :

Matière grasse (p. 100) Heiss

Matière grasse (p. 100) S.B.R.

\begin{tabular}{l|l|l|l|l|}
21,5 & 22,5 & 23,0 & 22,5 & 24,0 \\
21,9 & 22,5 & 23,8 & 22,8 & 24,7 \\
\hline
\end{tabular}

sur des Camemberts mis en vente en début d'affinage.

Prenons maintenant des Camemberts non consommables, parce que trop affinés et altérés, pour obtenir des écarts maximals :

Matière grasse (p. 100) Van Gülick

Matière grasse (p. 100) Heiss

Matière grasse (p. 100) S.B.R.

\begin{tabular}{l|l|l|l}
26,8 & 18,3 & 27,0 & 19,0 \\
26,5 & 18,0 & 26,0 & 18,5 \\
26,0 & 17,4 & 25,7 & 18,8
\end{tabular}

et encore :

Matière grasse/E.S. (p. 100) Van Gülick

Matière grasse/E.S. (p. 100) Heiss

Matière grasse/E.S. S.B.R.

\begin{tabular}{l|l|l}
45,25 & 50,35 & 49,14 \\
39,46 & 44,94 & 44,90 \\
40,40 & 45,66 & 45,35
\end{tabular}

Pour illustrer ce que nous avons dit de la méthode Van Gülick, concernant les pourcentages de matière grasse d'autant plus forts 
que le fromage est plus affiné, comparons-les à ceux de la Heiss qui ne sont pas influencés par le degré de lipolyse :

\begin{tabular}{l|l|l}
\hline Camemberts, matière grasse p. 100 & Heiss & Van Gülick \\
\cline { 1 - 2 } Moyenne de 20 en début d'affinage & 20,54 & $21,97+1,43$ \\
Moyenne de 8, affinés & 21,48 & $23,42+1,94$ \\
Moyenne de 2, très affinés & 26,20 & $28,46+2,26$ \\
\hline
\end{tabular}

Nos analyses ont porté principalement sur des Camemberts parce que, parmi les fromages courants, ils sont de ceux qui comportent le pourcentage le plus élevé d'acides gras libres par rapport aux acides aliphatiques totaux [1], donc pouvant donner des écarts plus importants.

Ce n'est pas le cas pour les fromages de Hollande, par exemple ; la moyenne de six analyses donnant :

$\begin{array}{lll}\text { Matière grasse (p. 100) } & \text { S.B.R. 25,15 Van Gülick 25,46 }\end{array}$

Nous voulions donner les informations qui précèdent, car bon nombre de fromagers se fient uniquement aux dosages des matières grasses des fromages, au stade de la vente, faits par la méthode Van Gülick très largement diffusée depuis des dizaines d'années. De plus les résultats des laboratoires sont quelquefois mis en parallèles sur des échantillons qualifiés d'identiques. Les méthodes étant souvent différentes, on comprend mieux maintenant ces " querelles » d'experts.

Notons aussi que maintenant, dans le prix des laits à l'achat, la valeur de la matière grasse intervient pour 54,3 p. 100 et le lait écrémé pour 45,7 p. 100 . La matière grasse mise en œuvre dans les fabrications fromagères se retrouvant dans les rendements, la recherche de la perfection dans le taux final de gras/sec est moins importante que dans les années antérieures.

Nous pensons néanmoins qu'il est très important pour un fromager de bien connaître, par une méthode rapide, simple et peu onéreuse, le taux final de gras/sec dans les fabrications et ce, quel que soit le stade d'affinage du produit.

De plus, une telle méthode, donnant des résultats moins sujets à erreur que ceux de la Van Gülick, peut être utilisée avantageusement pour les analyses de routine ou de dépistage, étant entendu que, malgré des chiffres voisins de la S.B.R., cette dernière reste seule valable pour la confirmation d'une infraction. 


\section{Ré s u m é}

M. Desfleurs et B. Bailleul utilisent une modification de la méthode de Heiss pour la détermination rapide de la matière grasse des fromages qui donne des résultats plus justes que ceux de la Van Gülick quand les fromages sont affinés.

\section{Su m m a r y}

M. Desfleurs and B. Bailleul used modification of the Heiss' method for a quick determination of cheeses fat content with better results than Van Gülick with ripened cheeses.

Reçu pour publication le 15 mai 1974.

\section{Bibliographie}

[1] Adda (J.) et Dumont (J. P.) (1974). - Les substances responsables de l'arôme des fromages à pâte molle. Le Lait, t. LIV, $\mathrm{n}^{\circ}$ 531-532, janv.-fév., 1-21.

[2] ARRÊTÉ du 8 JANVIER 1970, J.O. du 25 janv. 1970.

[3] Cador (P.) et Macherel (P.) (1944). - Le Lait, t. XXIV, nº 237-238 et 239-240, juil.-sept. et oct.-déc., 196-209 et 289-310.

[4] Convention Internationale, J.O. du 31 août 1934. Norme Française homoloGUÉE V 04-286 par arrêté du 3 janv. 1971, J.O. du 12 janv. 1971.

[5] Graduation des butyromètres a lait. - Norme NF B 35-521. Contrôles par le Laboratoire National d'Essais. J.O. du 23 avril 1954.

[6] Heiss (E.) (1961). - Versuche Zur Bestimmung des Käsefettgehaltes mit Hilfe Schnellmethoden. Dtsch. Molkerei Ztg., 82 (3), 19 janv., 67-70.

[7] Jacquet (J.) et LenorR (J.) (1969). - Mécanismes intimes de l'affinage des fromages. Economie et Médecine Animales, n 1, janv.-fév., 38-71.

[8] Kuzdzal-Savore (S.) et Langlois (D.) (1972). - A propos de la méthode Gerber. La Technique Laitière, $\mathrm{n}^{\circ} 755$, oct., 13.

[9] Méthode Röse-Gottlieb, norme NF 04-214.

[10] Pien (J.) (1943), - L'application de la méthode Gerber et la pénurie des réactifs. L'Interprofession Laitière.

[11] Pien (J.), Désirant (J.) et Avril (P.) (1972). - Le dosage de la matière grasse des laits homogénéisés par la méthode Gerber. Ann, Fals. Exp. Chim., 385-402.

[12] Pien (J.) et Mile Maurice (G.) (1938). - Relations de densité dans les produits laitiers. Le Lait, t. XVIII, $\mathrm{n}^{\circ} 176$, juin, 582-610.

[13] Piget (J.) (1965). - Chromatographie des acides aliphatiques volatils sur papier et en phase gazeuse. Dip. d'études sup. de l'Université de Caen.

[14] Teichert (1934). - Teneur en graisse de la matière sèche des fromages. Molkerei Ztg., t. 48, $\mathrm{n}^{\circ}$ 72-75.

[15] Villette (O.), Jacouet (J.) et Letourneur (G.) (1959). - Contribution à l'étude de la maturation des fromages normands à pâte molle. C.R. Ac. Agr. de France, 9 déc. 